\title{
ALKENE BASED MONOLAYER FILMS AS ANTI-STICTION COATINGS FOR POLYSILICON MEMS
}

\author{
W. Robert Ashurst, Christina Yau, Carlo Carraro, Roger T. Howe*, and Roya Maboudian \\ Berkeley Sensor \& Actuator Center \\ Department of Chemical Engineering \\ * Departments of Electrical Engineering and Computer Sciences and Mechanical Engineering \\ University of California at Berkeley, Berkeley CA 94720-1492 USA \\ tel (510) 643-3489, fax (510) 642-4778, ashurbr@uclink4.berkeley.edu
}

\begin{abstract}
This paper describes a new class of anti-stiction coatings for silicon MEMS that is based on the free radical reaction of a primary alkene (e.g., 1-octadecene, $\mathrm{C}_{16} \mathrm{H}_{33} \mathrm{CH}=\mathrm{CH}_{2}$ ) with hydrogen terminated silicon $[1,2]$. The new monolayer coating has several key advantages over the previously reported octadecyltrichlorosilane (OTS) and $1 \mathrm{H}, 1 \mathrm{H}, 2 \mathrm{H}, 2 \mathrm{H}-$ perfluorodecyltrichlorosilane (FDTS) based self-assembled monolayers (SAMs) $[3,4]$ : 1) The coating does not produce $\mathrm{HCl}$ at any stage in the monolayer formation whereas chlorosilane-based chemistry does. 2) The coating does not require the formation of an intervening oxide layer. 3) The film formation procedure for alkene-based monolayers is simpler than for chlorosilane-based SAMs for two main reasons. First, the surface oxidation step is eliminated. Second, the coating solution does not need to be conditioned before use, since water is not a reagent in this process. 4) The coating process is much more robust since it is essentially insensitive to relative humidity. 5) The coated surfaces have much fewer particulates in comparison to those coated with OTS. 6) The coating process can be made selective to coat only exposed silicon by generating radicals using a radical initiator.

The coating is evaluated in several ways, including X-ray photoelectron spectroscopy (XPS), atomic force microscopy (AFM), contact angle analysis (CAA), work of adhesion by cantilever beam array (CBA) technique and coefficient of static friction using a sidewall testing device. The octadecene film is compared to the OTS SAM. XPS data confirm the absence of oxygen in both freshly prepared samples and in samples aged for four months in laboratory ambient. AFM shows that the samples which receive 1-octadecene films accumulate far fewer particles during processing than those which receive the OTS SAM treatment. l'hese improvements have been achieved without sacrificing the stiction characteristics of coated micromachines, in that water and hexadecane contact angles, apparent work of adhesion, and coefficient of static friction data are found to be similar to those of OTS.
\end{abstract}

\section{INTRODUCTION}

The large surface-area-to-volume ratios of surface and bulk micromachined micromechanisms bring the role of stiction into the foreground, as adhesion of these mechanisms to adjacent surfaces is a major failure mode for MEMS [5-7]. Stiction is a term that has been applied to the unintentional adhesion of compliant microstructure surfaces when restoring forces are unable to overcome interfacial forces such as capillary, van der Waals and electrostatic attractions. Release stiction, the adhesion of surfacemicromachined structures to the underlying substrate following the final sacrificial layer etch, is caused primarily by liquid capillary forces. Engineering solutions to this problem include a variety of techniques which have been reviewed elsewhere [5-7]. Most of these techniques, however, do not prevent adhesion from occurring during micromachine operation. Microstructure surfaces may come into contact unintentionally through acceleration or electrostatic forces, or intentionally in applications where surfaces impact or shear against each other. When adhesive attractions exceed restoring forces, surfaces permanently adhere to each other causing device failure -- a phenomenon known as in-use stiction. In addition, it is known that on the microscale, friction is strongly dependent upon adhesion [8].

In order to alleviate these adhesion-related problems, both the topography and the chemical composition of the contacting surfaces must be controlled. Several approaches have been developed to address these tribological problems and have been reviewed elsewhere [5-7]. Our group has investigated both OTS and FDTS SAMs for alleviating adhesion in polycrystalline silicon microstructures. These SAMs have been shown to effectively eliminate release stiction, and significantly reduce in-use stiction, friction, and to some extent wear [9-11].

Although these SAM coatings have been shown to significantly reduce both release and in-use stiction, they possess a number of limitations intrinsically related to their chemistry. As with any chlorosilane-based SAM precursor molecule, the first step in the reaction sequence for binding the molecule to the substrate is the hydrolysis of one or more $\mathrm{Si}-\mathrm{Cl}$ bonds. This hydrolysis thus results in one equivalent of $\mathrm{HCl}$ for each $\mathrm{Si}-\mathrm{Cl}$ bond that is hydrolyzed. The presence of $\mathrm{HCl}$ in the SAM coating solution poses a threat to exposed aluminum interconnects, as well as other metals or metal compounds that may be present. Additionally, silicon surfaces must first be oxidized in order for any chlorosilane SAM to form. This is usually accomplished by growing a thin chemical oxide $(\sim 20 \AA)$ with an oxidizing agent such as hydrogen peroxide. This oxidation step further complicates the coating process and poses a barrier for any strategy to achieve selective coatings. Another limitation arises from the ability of the precursor molecule to polymerize. As long as the precursor molecule has a functionality greater than one, bulk polymerization can occur, producing particulates that are up to several microns in diameter. These particulates can mechanically interfere with the device operation and pose reliability concerns. Unfortunately, there is no satisfactory method for removal of the polymerized clusters once they have agglomerated on the surfaces of the micromachines.

Other limitations of the chlorosilane SAM coatings arise from the coating procedure. The coating process is somewhat cumbersome in that the SAM solution must be freshly made and appropriately conditioned immediately before each coating. This is due to the sensitivity of the SAM solution to ambient humidity, and the ability of the SAM precursors to polymerize. As mentioned earlier, a hydrolysis step is required for SAM formation. The water, which is a reagent, necessary for this step is introduced to the organic SAM solution by mass transfer from the 
ambient air. Hence it is necessary to control the ambient humidity such that there will be enough water present in solution to carry out the hydrolysis, but an insufficient concentration to promote bulk polymerization of the SAM precursor molecules. The tolerable relative humidity range is from $30 \%$ to $70 \%$.

Based upon the desired anti-stiction film characteristics, in particular surface hydrophobicity, and the considerations of the chemistry and coating processes, we have investigated the feasibility of employing a new class of monolayer coatings as antistiction films. This new class of films abandons the chlorosilane chemistry and adopts a free radical reaction of a primary alkene to bind the precursor molecule to a hydrogen terminated silicon substrate $[\mathbf{1 , 2}]$. The reagent we have examined is 1-octadecene, $\mathrm{C}_{16} \mathrm{H}_{33} \mathrm{CH}=\mathrm{CH}_{2}$. Due to the substantially different chemistry of this precursor molecule, no $\mathrm{HCl}$ is produced during the reaction. Additionally, there is no need for oxidation of the surface, since the molecule binds directly to the silicon. Water is not a reagent in this reaction sequence, and hence the coating procedure is insensitive to ambient humidity. The alkene is very stable at room temperature; the coating solution can be stored indefinitely. The coating solution can even be re-used or recycled from previous treatments if it is so desired.

The coating procedure is described in detail below. We also report on the characteristics of the resulting film, using XPS, AFM, and CAA. Furthermore, wc have determined the values of work of adhesion by cantilever beam array technique, as well as coefficient of static friction and thermal stability in vacuum and oxidizing environments.

\section{EXPERIMENTAL}

The films studied in this work are the l-octadecene monolayer and the OTS SAM for comparison. Both precursor molecules contain a straight 18 -carbon chain, and bind to the substrate at only one end. Hence, the films created with these particular molecules are directly comparable in terms of antistiction behavior. For reference, structures were also prepared without any anti-stiction coating, i.e., oxidized surfaces. The procedure used for the OTS SAM coating was outlined in ref. 9 with the following exceptions. The solvent for the OTS was anhydrous iso-octane (Aldrich Chemical Co.) and the SAM formation was carried out for 60 minutes instead of 15 minutes. The procedure that was carried out for the oxidized microstructures consisted of a standard release followed by surface oxidation with $30 \% \mathrm{H}_{2} \mathrm{O}_{2}$ at $90{ }^{\circ} \mathrm{C}$ for 10 minutes. The structures were then rinsed with deionized water and either supercritically dried or removed directly from water and air-dried.

In order to address the issue of large volumes of waste solvent generated by the displacement rinses commonly found in monolayer preparation procedures, a solvent "fill/drain" approach was developed instead of an aspiration approach. A more complete description of this system can be found in ref. 12. This device consists of a Teflon FEP separatory funnel modified by cutting the top such that the rounded portion is removed. In addition, a Teflon ETFE pluy is machined to fit inside the modified funnel so as to leave sufficient volume above the cone for process liquids. The plug reduces the amount of solvents needed, by reducing the stagnant volume of the funnel. This device was used for all displacement rinses.

The general coating procedure for the alkene-based films is summarized in Table 1. As with any microstructure release proccdurc, the first step is the sacrificial layer etch. Typically, this is done with concentrated HF for an amount of time that depends of the fabrication history of the structures being released. After the sacrificial layer etch, the silicon surfaces will be hydrogen terminated. However, the etch products that result from removal of the sacrificial layers need to be thoroughly rimsed away, and the etch solution displaced. At this point, a deionized water rinse (18$\mathrm{M} \Omega-\mathrm{cm}$ ) serves both of these purposes. However, during the time that the rinsing is taking place, the water can oxidize the hydrogen terminated surface to the extent that the integrity of the alkene coating may be jeopardized. Since the hydrogen termination is necded for the reaction, the silicon surfaces are treated again with $\mathrm{HF}$ to recover the hydrogen termination. This time, however, the HF is displaced with isopropyl alcohol (IPA) which does not oxidize hydrogen-terminated silicon. Additionally, the IPA serves as a useful intermediate between the organic 1-octadecene coating solution and the aqueous HF. The structures are then rinsed with the 1-octadecene. Now the structures are ready to be transferred into a reactor containing the octadecene coating solution. This solution is a 9:1 v/v solution of hexadecane:1-octadecene. The hexadecane is anhydrous spectroscopic grade (Aldrich Chemical Co.) and the 1-octadecene is technical grade, $90 \%$, anhydrous (Aldrich Chemical Co.). The octadecene is diluted to reduce the potential for bulk polymerization of the difunctional alkene. After the structures are placed inside the reactor and the lid is sealed, a dry nitrogen gas is turned on in order to purge oxygen from the vapor space above the coating solution. This gas flow was maintained throughout the heating process. The reactor is heated to $180^{\circ} \mathrm{C}$ and held at temperature for 30 minutes. Heating was chosen as the method to generate free radicals; this is the step during which the monolayer forms. The reactor is then allowed to cool. The structures are then removed from the coating solution and thoroughly displacement rinsed into petroleum ether (Fisher, b.p. $38-54^{\circ} \mathrm{C}$ ). The structures are then displaced into IPA and rinsed further. Next, the IPA is completely washed away with DI water, while the liquid surface is aspirated. At this point, the structures can be removed from the water.

\begin{tabular}{|c|c|c|}
\hline Step & Procedure & $\begin{array}{c}\text { Approximate } \\
\text { Time }\end{array}$ \\
\hline $\begin{array}{c}\text { Sacrificial } \\
\text { Layer Etch }\end{array}$ & HF & $10 \mathrm{~min}$. \\
\hline $\begin{array}{c}\text { Octadecene } \\
\text { Coating }\end{array}$ & Water rinse to pH neutrality & $10 \mathrm{~min}$. \\
\cline { 2 - 3 } & $\begin{array}{c}\text { Secondary HF for hydrogen } \\
\text { termination }\end{array}$ & $2 \mathrm{~min}$. \\
\cline { 2 - 3 } & Displacement rinse to IPA & $5 \mathrm{~min}$. \\
\cline { 2 - 3 } & $\begin{array}{c}\text { Displacement rinse to } 1- \\
\text { octadecene solution }\end{array}$ & $5 \mathrm{~min}$. \\
\cline { 2 - 3 } & 1-Octadecene treatment at $180^{\circ} \mathrm{C}$ & $30 \mathrm{~min}$. \\
\hline \multirow{4}{*}{$\begin{array}{c}\text { Rinse and } \\
\text { Dry }\end{array}$} & $\begin{array}{c}\text { Displacement rinse to petroleum } \\
\text { ether }\end{array}$ & $5 \mathrm{~min}$. \\
\cline { 2 - 3 } & Displacement rinse to IPA & $5 \mathrm{~min}$. \\
\cline { 2 - 3 } & Displacement rinse to water & $10 \mathrm{~min}$. \\
\cline { 2 - 3 } & Removal from water & \\
\hline
\end{tabular}

Table 1. The basic processing steps for a 1-octadecene antistiction coating.

Contact angle data were taken with DI water (resistivity 18$\mathrm{M} \Omega-\mathrm{cm}$ ) and spectroscopic grade hexadecane (Aldrich Chcmical Co.) according to the sessile droplet method with a Ramé Hart 100 A goniometer. Droplet size was approximately $4 \mu \mathrm{l}$, and the measurement reproducibility was $\pm 2^{\circ}$. A Digital Instruments Nanoscope III atomic force microscope was used to image sample surfaces and quantify their roughnesses. XPS data were taken with a Kratos XSAM 800 system. The probing system used for all micromachinc actuation was a Lucas-Signatone $\mathrm{S}-1160$ probe station with a Mitutoyo FineScope 60 microscope, equipped with a Sony CCD-IRIS camera. A Nomarski style differential interference contrast prism (manufactured by Olympus) was used with a Mitutoyo M Plan Apo 10x objective on the microscope. 
All micromachine actuation was done under normal laboratory ambient conditions, $20^{\circ} \mathrm{C}$ and $50 \%$ relative humidity. Work of adhesion by cantilever beam array technique was performed in accordance with the procedure outlined in ref. 9. The CBA test structures used in this study were fabricated at Sandia National Labs, Albuquerque, NM. The beams were of lengths varying from 150 to 1700 microns, 2.5 microns thick and 2 microns above the substrate. The RMS roughness of the groundplane underneath the beams was about $2.3 \mathrm{~nm}$. Coefficient of static friction was determined from a sidewall testing device described in ref, 10. Actuation of this device and analysis of data were in accordance with the procedures outlined in ref. 13 .

The thermal stability of the octadecene film has been studied on $\mathrm{Si}(100)$ in vacuum and in oxidizing ambients. In the latter case, the sample was exposed to high temperature, in laboratory air, for five minutes, and then removed to a room temperature aluminum slab for cooling. The five minute exposure time was chosen based upon estimates of integrated circuit packaging times. The film stability was checked by contact angle goniometry, and the water contact angles were taken immediately after the samples cooled.

\section{RESULTS AND DISCUSSION}

Contact angle data are summarized in Table 2. Also listed are the representative values for oxide and OTS coated surfaces for reference. The contact angle data indicate that the molecular packing of film produced by the octadecene coating process is not as high as that produced by the OTS coating process. However, this difference appears to have little effect on the anti-stiction properties of the film. XPS analysis on $\mathrm{Si}(100)$ confirmed the absence of silicon oxide in the freshly made 1-octadecene samples. To gauge the passivation effect of the coating, samples were prepared and left in laboratory environment for various durations. Even after four months of storage, no silicon oxide was observed with XPS.

Although it has been observed that the octadecene reaction is not selective if the initiation is by heating, it can be made selective to hydrogen terminated silicon by changing the method of free radical formation. Ultraviolet excitation and radical initiators (such as 2,2'-azobisisobutyronitrile) are two other ways in which free radicals may be formed. Ultraviolet excitation may not be an appropriate means of initiation due to conformality concerns for micromachines. It has been observed that the use of a radical initiator results in a selective coating process. Although not selective, heating remains the simplest and most uniform means of causing the monolayer formation and hence, all data reported here arises from monolayers formed by this method.

AFM was used to study particulates that accumulate on the surface during the coating procedure. Figures 1 (a) and (b) are AFM images for OTS and 1-octadecene on $\mathrm{Si}(100)$ respectively. These samples have been treated alongside microstructures, and hence, undergone every processing step exactly as the structures. They were not cleaned in any way after final removal from water. The scan size is $30 \mu \mathrm{m} \times 30 \mu \mathrm{m}$ and the $z$ scale (height) is $100 \mathrm{~nm}$. Note that in contrast to octadecene, the OTS sample is cluttered with large particulates that are on the order of microns in diameter. The RMS roughness based on image statistics is $25 \mathrm{~nm}$ for the OTS sample and $0.4 \mathrm{~nm}$ for the octadecene sample.

In CBA test structures, beams as long as 900 microns were consistently found to be free standing after release. The CBAs were subsequently used to find apparent work of adhesion values for the films. Due to the inherent variation from chip to chip, beam sticking probability data were accumulated over many samples prepared independently by two operators. The OTS sticking probability function exhibits a sharp increase in the fraction stuck as a function of beam length near the detachment length, whereas the octadecene curve exhibits a more gradual transition. More work is underway to determine the nature of the difference between these coatings in this respect. The average values of apparent work of adhesion are summarized in Table 2 . Note that the work of adhesion for the octadecene coating is similar to that of the OTS SAM. Additionally, the work of adhesion for both coatings is roughly four orders of magnitude lower than that of the standard oxide coating.

Sidewall friction testers were used to determine the coefficient of static friction on micromachine sidewalls. Again, due to chip to chip variation, a number of samples prepared by different operators were tested for each surface treatment. The results for $\mu_{\mathrm{s}}$ are summarized in Table 2 . Note that the 1 octadecene treatment and the OTS SAM have similar coefficients of friction, and that their values are roughly a factor of 20 less than that of an oxidized surface.

\begin{tabular}{|c|c|c|c|c|}
\hline $\begin{array}{c}\text { Surface } \\
\text { Coating }\end{array}$ & $\begin{array}{c}\text { Water } \\
\text { Contact } \\
\text { Angle }\end{array}$ & $\begin{array}{c}\text { Hexadecane } \\
\text { Contact } \\
\text { Angle }\end{array}$ & $\begin{array}{c}\text { Work of } \\
\text { Adhesion } \\
\left(\mathrm{mJ} / \mathrm{m}^{2}\right)\end{array}$ & $\mu_{\mathrm{S}}$ \\
\hline Octadecene & $104^{\circ}$ & $35^{\circ}$ & 0.009 & 0.050 \\
\hline OTS & $109^{\circ}$ & $38^{\circ}$ & 0.012 & 0.073 \\
\hline Oxide & $0-30^{\circ}$ & $0-20^{\circ}$ & 30 & 1.1 \\
\hline
\end{tabular}
preparations.

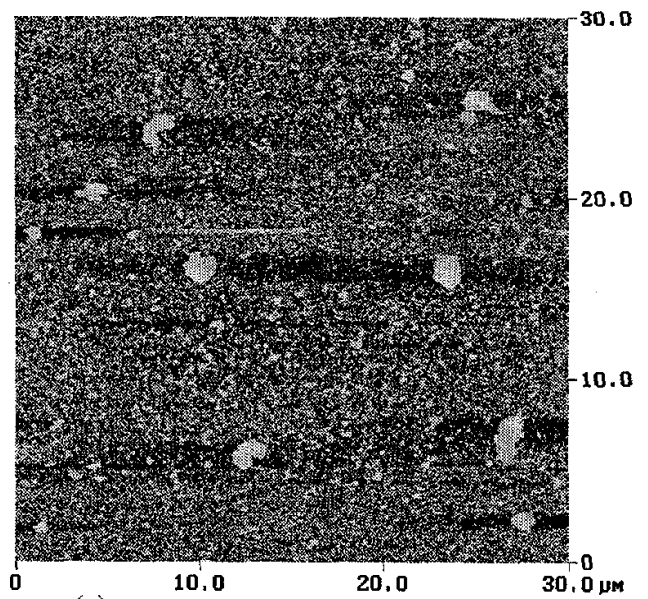

(a)

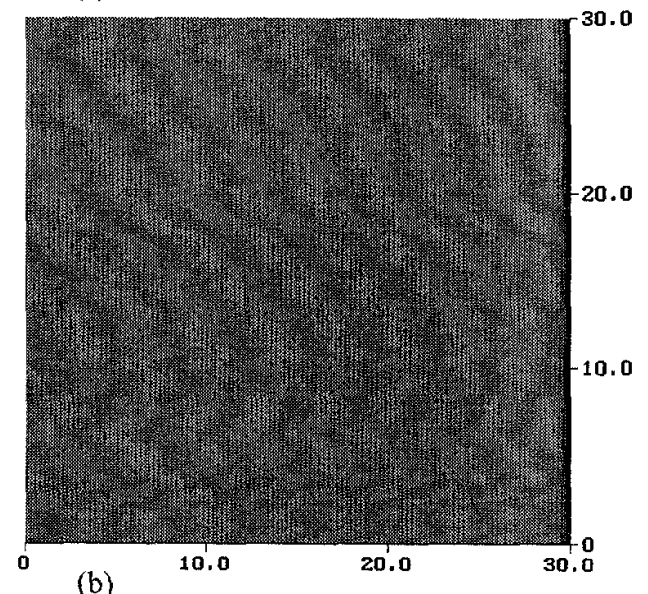

(b)

Figure 1: AFM image of (a) an OTS SAM and (b) 1-octadecene coated Si(100) chip. The samples have undergone identical treatments as micromachine chips. The particulates on the OTS surface are attributed to bulk polymerization of the precursor 
molecules. The z scale in both images is $100 \mathrm{~nm}$ and the RMS roughness is $25 \mathrm{~nm}$ for (a) and $0.4 \mathrm{~nm}$ for (b).

Thermal behavior of the octadecene monolayer in vacuum has been studied by Sung et al. [2]. The film was found to degrade in vacuum at about $350^{\circ} \mathrm{C}$, and by $525^{\circ} \mathrm{C}$ evidence of a thin $\mathrm{SiC}$ film was observed. The same authors have investigated the thermal stability of the OTS coating. The film degradation begins at about $450{ }^{\circ} \mathrm{C}$, and by about $620^{\circ} \mathrm{C}$, only the underlying oxide remains [2]. In air, the thermal stability is approximately the same for both the octadecene and OTS films. Both films begin to degrade at about $225^{\circ} \mathrm{C}$, where the octadecene film shows a sharper falloff in contact angle than does the OTS film. Note that the FDTS films were found to be stable in air up to $400{ }^{\circ} \mathrm{C}$ [9].

\section{CONCLUSIONS AND FUTURE WORK}

Based upon the data presented here, we find that the antistiction properties of films produced with the alkene chemistry are indeed comparable to those produced with the trichlorosilane SAMs, but without many of the limitations imposed by the chlorosilane chemistry. The apparent work of adhesion for 1octadecene coated polysilicon is nearly four orders of magnitude lower than that of the standard oxide coating and slightly less than that of the OTS SAM. The sidewall friction testers showed that the octadecene treatment and the OTS SAM have similar coefficients of static friction, and that they are roughly a factor of 20 less than that of the standard oxidized surface. XPS data confirms that the alkene-based monolayers are effective at passivating the silicon surface to oxidation. It was also shown that films produced with alkene precursors accumulate fewer particulates than those produced by the OTS precursor. The thermal stability of the octadecene films is nearly the same as that for the OTS SAM.

Thermal annealing of octadecene films in vacuum has been shown to produce silicon carbide [2]. Current work suggests that an octadecene treatment followed by rapid thermal annealing in vacuum could become a viable route to further carbidization by some other method.

\section{ACKNOWLEDGEMENTS}

The authors gratefully acknowledge the financial support of the National Science Foundation (grant DMI-9908169) and Sandia National Labs. Additionally, the authors wish to thank Drs. Mike Dugger, Maarten deBoer, and Jeff Sniegowski of Sandia for providing micromachine test structures and for many valuable discussions. The XPS data were obtained by Christina Lee and Dr. Jon Kluth. Additional financial support from the National Science Foundation in the form of a graduate fellowship (WRA) is gratefully acknowledged.

\section{REFERENCES}

1. M. R. Linford, P. Fenter, P. M. Eisenberger, and C. E. D. Chidsey, "Alkyl Monolayers on Silicon Prepared from 1-Alkenes and Hydrogen-Terminated Silicon", J. Am. Chem. Soc. 117, 31453151 (1995).

2. M. M. Sung, G. J. Kluth, O.W. Yauw, and R. Maboudian, "Thermal Behavior of Alkyl Monolaycrs on Silicon Surfaces", Langmuir, vol. 13, no 23, 6164-6168 (1997).

3. M. R. Houston, R. T. Howe, and R. Maboudian, "SelfAssembled Monolayer Films as Durable Anti-Stiction Coatings for Polysilicon Microstructures", Technical Digest of the 1996 Solid-
State Sensor and Actuator Workshop, Hilton Head '96, pp. 42-47 (1996).

4. U. Srinivasan, M. R. Houston, R. T. Howe, and R. Maboudian, "Self-Assembled Fluorocarbon Films for Enhanced Stiction Reduction", Technical Digest, Proceedings of the 9th International Conference on Solid-State Sensors and Actuators, Transducers '97, pp. 210-213 (1997).

5. R. Maboudian and R.T. Howe, "Critical review: Adhesion in surface micromechanical structures," J. Vac. Sci. Technol. B, vol. 15, pp. 1-20, (1997); and references therein.

6. N. Tas, T. Sonnenberg, H. Jansen, R. Legtenberg, and M. Elwenspoek, "Stiction in surface micromachining", $J$ Micromech Microeng, vol. 6, pp. 385-397, (1996); and references therein.

7. K. Komvopoulous, "Surface Engineering and Microtribology for Micromechanical Systems", Wear, vol. 200, pp. 305-327, (1996).

8. B. Bhushan, Tribology and Mechanics of Magnetic Storage Devices, Springer, New York, (1990).

9. U. Srinivasan, M. R. Houston, R. T. Howe, and R. Maboudian, "Alkyltrichlorosilane-Based Self Assembled Monolayer Films for Stiction Reduction in Silicon Micromachines" J. Microelectromechanical Systems, vol. 7, no. 2, (1998).

10. D. C. Senft and M. T. Dugger, "Friction and Wear in Surface Micromachined Tribological Test Devices", SPIE vol. 3224, pp. 31 -38 , (1997).

11. U. Srinivasan, J. D. Foster, U. Habib, R. T. Howe, and R. Maboudian, "Lubrication of Polysilicon Micromechanisms with Self-Assembled Monolayers", Technical Digest of the 1998 SolidState Sensor and Actuator Workshop, Hilton Head '98, pp. 156-161 (1998).

12. R. Maboudian, W. R. Ashurst, and C. Carraro, "SelfAssembled Monolayers as Anti-Stiction Coatings for MEMS: Characteristics and Recent Developments", Sensors and Actuators $A$, in press.

13. W. R. Ashurst, C. Yau, C. Carraro, M. T. Dugger, R. T. Howe, and R. Maboudian, Manuscript in preparation. 